\title{
The Construction and Genetic Analysis of Polyploids and Aneuploids of the Pentose-fermenting Yeast, Pachysolen tannophilus
}

\author{
By ALLEN P. JAMES* AND DIANA M. ZAHAB \\ Division of Biological Sciences, National Research Council of Canada, 100 Sussex Drive, Ottawa, \\ Ontario KIA 0R6, Canada
}

(Received 30 November 1982)

Triploid and aneuploid strains of the xylose-fermenting yeast Pachysolen tannophilus were constructed. This species is a strongly homothallic organism in which the haploid phase normally predominates. The technique for producing polyploids involved prototrophic selection and interruption of the normal sequence of events leading from nuclear fusion to meiosis. Confirmation of triploidy was obtained by tetrad analysis. The haploid chromosome number of $P$. tannophilus was estimated to be between 5 and 7 .

\section{INTRODUCTION}

It has recently been observed that the yeast Pachysolen tannophilus is capable of fermenting xylose as well as hexoses to alcohol (Schneider et al., 1981 ; Slininger $e$ t al., 1982). Such organisms are of practical as well as intrinsic interest because they provide one possible means of widening the range of substrates available for the conversion of biomass to ethanol; xylose is a major component of many cellulosic materials but up to now has remained unused in industrial fermentation processes.

Most if not all the strains of Saccharomyces cerevisiae used in commercial alcohol production are polyploid or possibly aneuploid, from which it can be conjectured that supernumerary chromosomes are, for one reason or another, advantageous. Can strains of higher ploidy be constructed in $P$. tannophilus? And if so, is alcohol production from xylose in any way affected? This paper describes the successful construction of both triploid and aneuploid strains of this organism. Together with haploids and diploids already available (James \& Zahab, 1982), they provide a ploidy series for studying the effect of gene or chromosome dosage on xylose fermentation. The results of this latter study are presented in a companion paper (Maleszka et al., 1983).

\section{METHODS}

Organism. Two strains of Pachysolen tannophilus were used, NRRL Y2461 and NRRL Y2463. All mutant strains were derived from these two haploids.

Media. The standard yeast extract/peptone/dextrose agar medium (YEPD) was used for general purposes. This medium contains $10 \mathrm{~g}$ yeast extract, $20 \mathrm{~g}$ peptone, $20 \mathrm{~g}$ dextrose and $20 \mathrm{~g}$ agar per litre. For sporulation, the medium was yeast extract/malt extract agar medium (YM) which contains $4 \mathrm{~g}$ yeast extract, $10 \mathrm{~g}$ malt extract, $4 \mathrm{~g}$ dextrose, and $200 \mathrm{~g}$ agar per litre. The $\mathrm{pH}$ was adjusted to 5.0 prior to autoclaving.

For genetic characterization, appropriately supplemented yeast nitrogen base agar media (YNB) were used.

Genetic analyses. Matings were made as follows. Cells of the two auxotrophic parent strains were mixed as a streak on $\mathrm{YM}$ and incubated at $30^{\circ} \mathrm{C}$. At $3 \mathrm{~d}$ the mixture was picked, streaked widely on the appropriate YNB selective agar medium, and reincubated at $30^{\circ} \mathrm{C}$. Prototrophic colonies could be isolated at $4 \mathrm{~d}$.

Strains were sporulated by streaking cells on YM medium and incubating for $4 \mathrm{~d}$ at $30^{\circ} \mathrm{C}$.

Standard methods were used for tetrad analysis except that pretreatment with cytohelicase is not necessary; tetrads are released from their ascophores as they mature, and become free-floating. Dissection of spores is more easily accomplished, however, if a weak solution of cytohelicase is added to the spore culture at the time of streaking for manipulation. 


\section{RESULTS AND DISCUSSION}

Polyploids of yeast are commonly restricted to heterothallic species and are usually obtained through the use of spontaneously occurring diploids that are homozygous at the mating locus, aa or $\alpha \alpha$. Such strains mate readily with haploids of the opposite mating type (aa $\times \alpha$, or $\alpha \alpha \times$ a) to produce triploids, or with themselves (aa $\times \alpha \alpha)$ to produce tetraploids.

The life cycle of $P$. tannophilus is not conducive to such techniques because it is a strongly homothallic organism in which the haploid is predominant. In fact, the diplophase is normally confined to a transient state in which a diploid nucleus, produced by fusion of the products of mitosis, immediately undergoes meiosis to restore the haploid condition. Nevertheless, two relatively simple techniques can be used to procure stable diploid cultures (James \& Zahab, 1982). One of these consists, essentially, of cloning the diploid nucleus by interrupting the sequence of events leading to ascospore formation. This procedure results in homozygous diploid strains. The other method involves the same general procedure but includes prototrophic selection to produce heterozygous diploids. The frequency of hybridization is low, about $10^{-7}$, but entirely adequate for genetic purposes.

A possible method of constructing polyploid strains is implicit in the rather unorthodox mating behaviour of this yeast. No evidence of stable mating types has been detected as yet; a few haploid segregants are recalcitrant to hybridizing with any other haploid, but none has displayed any propensity for preferential 'mating' with any other. It was this property of unrestricted hybridization that suggested that polyploids might be constructed through the simple expedient of mating haploids to diploids or diploids to diploids. This possibility was tested using the haploids and diploids shown in Table 1. The procedure was apparently successful, and prototrophic colonies were obtained with frequencies that were comparable to those obtained by the mixing of auxotrophic haploids to produce diploids.

\section{Haploid $\times$ diploid}

Eight presumptive triploids from each of two different crosses, P39 and P56, (see Table 1) were sporulated and subjected to tetrad analysis. Sporulation was poor in most of these, but sufficient tetrads were isolated to provide the summarized data of Tables 2 and 3. Several features of the data are of particular interest.

(1) It is evident that the zygotic isolates are not identical within either of the two groups; there are sharp differences in the frequencies with which their spores produced viable clones. (2) There are significant differences between isolates in the segregation ratios of the marker genes. Although some of these cultures may be triploid, others are clearly aneuploid and a few, P39-\#3 in particular, may well be diploid. The frequency of spore germination in this isolate was high and the segregational data suggest that its chromosomes could have been derived from only two of its supposed three parents, P31-9C and P17-1A. (3) The frequency of spore germination among the potential triploids was much lower than that expected of diploids, but was nevertheless surprisingly high. It was, in fact, about ten times higher than that found with triploids of $S$. cerevisiae. Reduced viability of triploid segregants is usually attributed to metabolic imbalance imposed by aneuploidy induced at meiosis. It is evident that if any of these strains of $P$. tannophilus is indeed triploid then either this organism has fewer chromosomes than $S$. cerevisiae or else it is much more tolerant of chromosome imbalance.

A low frequency of spore viability is inimical to tetrad analysis and the segregational data of Tables 2 and 3 are therefore presented in the form of random spore analysis, a procedure which utilizes more data at the expense of a possible introduction of biases due to differential survival of genotypes. Assuming trivalent pairing in triploids, the expected segregation of a $+1-1-$ genotype is $1: 1(+:-)$. That of a $+/+/-$ genotype is $4 \cdot 4: 1(+:-)$. Neither ratio is affected by centromere linkage. Accumulated data are compared with these expectations at the bottom of each table, omitting those instances (bracketed) in which the ratios are at obvious variance with expectation. Agreement is good, and it may be concluded from this table that the isolates include strains that, if not triploid, are at any rate trisomic for at least one or two chromosomes, lys 1 and arg 1 being linked (James \& Zahab, 1982). 
Table 1. Genotypes of haploid and diploid strains of P. tannophilus and expected genotypes of polyploids derived from them

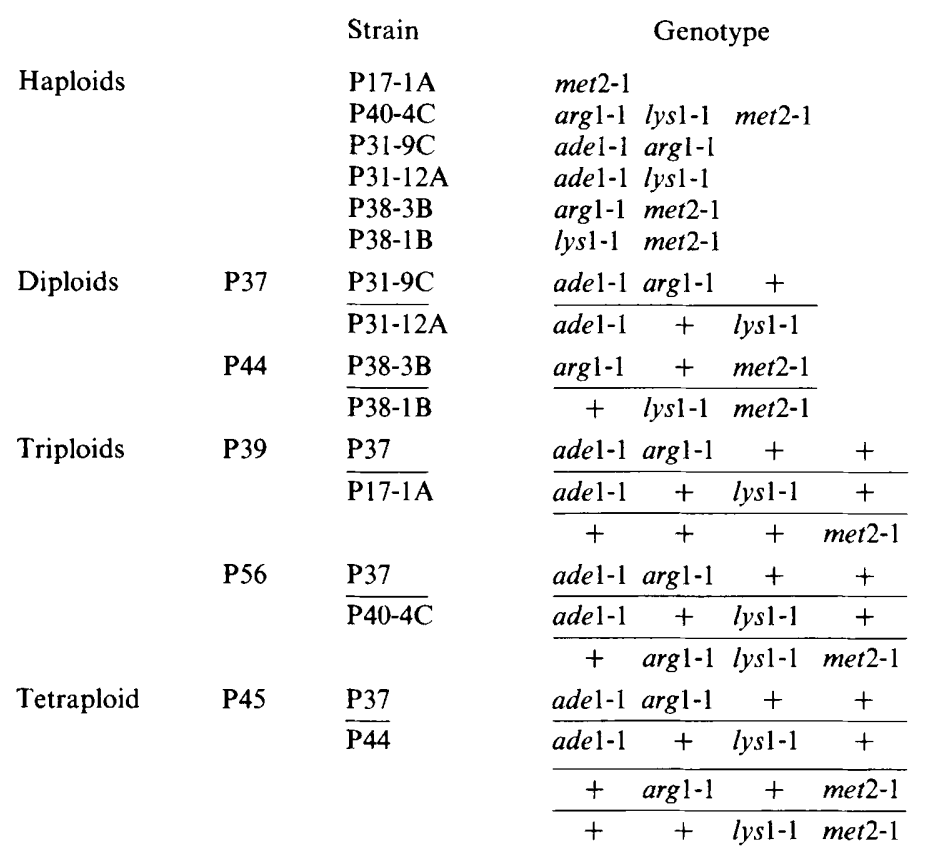

Table 2. Random-spore analyses of tetrad segregations of isolates of the presumptive triploid P39, ade arg ++ lade + lys $+1+++$ met

$+:-$ ratios that are in obvious conflict with expectation are in round brackets.

\begin{tabular}{|c|c|c|c|c|c|c|c|c|c|c|}
\hline \multirow[b]{2}{*}{ Isolate } & \multirow{2}{*}{$\begin{array}{c}\text { Spore } \\
\text { viability }\end{array}$} & \multirow{2}{*}{$\begin{array}{c}\text { Complete } \\
\text { tetrads }\end{array}$} & \multicolumn{2}{|c|}{$\underbrace{}_{\text {Ade }}$} & \multicolumn{2}{|c|}{ Arg } & \multicolumn{2}{|c|}{ Lys } & \multicolumn{2}{|c|}{ Met } \\
\hline & & & + & - & + & - & + & - & + & - \\
\hline$\# 1$ & 0.33 & $0 / 16$ & 7 & 14 & 15 & 6 & 19 & 2 & 16 & 5 \\
\hline$\# 2$ & 0.79 & $7 / 14$ & 22 & 22 & $(21$ & 23) & $(44$ & 0) & 33 & 11 \\
\hline$\# 3$ & 1.00 & $14 / 14$ & 28 & 28 & (29 & 27) & (56 & 0) & (28 & 28) \\
\hline$\# 4$ & 0.39 & $9 / 16$ & 20 & 19 & (39 & $0)$ & (20 & 19) & (21 & 18) \\
\hline$\# 5$ & $0 \cdot 15$ & $1 / 27$ & 4 & 12 & 14 & 2 & 12 & 4 & 13 & 3 \\
\hline$\# 6$ & 0.22 & $1 / 29$ & 15 & 11 & 21 & 5 & 21 & 5 & 19 & 7 \\
\hline$\# 7$ & $0 \cdot 22$ & $2 / 30$ & 13 & 13 & 21 & 5 & 19 & 7 & 18 & 8 \\
\hline$\# 8$ & $0 \cdot 29$ & $0 / 31$ & 17 & 18 & 33 & 2 & 27 & 8 & 23 & 12 \\
\hline tal & & & 126 & 138 & 104 & 20 & 98 & 26 & 122 & 46 \\
\hline pected & nt pair & & 132 & 132 & 101 & 23 & 101 & 23 & 137 & 31 \\
\hline
\end{tabular}

Table 3. Random-spore analysis of tetrad segregations of isolates of the presumptive triploid P56, ade arg $++/$ ade + lys $+1+$ arg lys met

\begin{tabular}{|c|c|c|c|c|c|c|c|c|c|c|}
\hline \multirow[b]{2}{*}{ Isolate } & \multirow[b]{2}{*}{$\begin{array}{c}\text { Spore } \\
\text { viability }\end{array}$} & \multirow[b]{2}{*}{$\begin{array}{c}\text { Complete } \\
\text { tetrads }\end{array}$} & \multicolumn{2}{|c|}{ Ade } & \multicolumn{2}{|c|}{ Arg } & \multicolumn{2}{|c|}{ Lys } & \multicolumn{2}{|c|}{ Met } \\
\hline & & & + & - & + & - & + & - & + & - \\
\hline$\# 1$ & 0.35 & $0 / 15$ & 13 & 8 & 11 & 10 & 10 & 11 & 16 & 5 \\
\hline$\# 2$ & 0.11 & $1 / 16$ & 3 & 4 & 3 & 4 & 4 & 3 & 5 & 2 \\
\hline$\# 3$ & 0.44 & $2 / 16$ & 13 & 15 & 11 & 17 & 9 & 19 & 21 & 7 \\
\hline$\# 4$ & $0 \cdot 10$ & $0 / 15$ & 3 & 3 & 4 & 2 & 2 & 4 & 4 & 2 \\
\hline$\# 5$ & $0 \cdot 13$ & $1 / 16$ & 3 & 5 & 3 & 5 & 3 & 5 & 4 & 4 \\
\hline$\# 6$ & 0.58 & $3 / 16$ & 17 & 20 & 22 & 15 & 20 & 17 & 28 & 9 \\
\hline$\# 7$ & 0.77 & $7 / 12$ & 23 & 14 & 19 & 18 & 18 & 19 & 31 & 6 \\
\hline$\# 8$ & 0.19 & $1 / 16$ & 5 & 7 & 7 & 5 & 6 & 6 & 9 & 3 \\
\hline & & & 80 & 76 & 80 & 76 & 72 & 84 & 118 & 38 \\
\hline pected & t pairit & & 78 & 78 & 78 & 78 & 78 & 78 & 127 & 29 \\
\hline
\end{tabular}


The frequency with which spores of the possible triploids germinated to produce visible colonies, though low, was sufficiently high to permit the presence of triploids to be confirmed by genetic means. The method made use of those segregations in which all four meiotic products produced visible spore colonies (viable tetrads), and was based on the following rationale. The four viable tetrads produced by a trisomic or multiple trisomic can be expected to include monosomics or multiple monosomics. These may or may not proceed to a first-generation meiosis, but if they do they can at best produce only two viable segregants per tetrad. In contrast, the viable tetrads produced by a triploid will include not only multiple monosomics but also, on occasion, two normal diploids $(2 n)$ and two normal haploids $(n)$. The diploids can be expected to proceed through a normal first-generation meiosis to yield four viable segregants. In the absence of any unscheduled chromosome replication, the existence of these zero-generation diploids provides positive evidence of triploidy.

Five segregations conformed to the above requirements, each producing two spore colonies capable of producing rare conjugant asci, an indication of haploidy, and, as well, two spore colonies capable of producing many non-conjugant asci, an indication of diploidy. The latter, when subjected to tetrad analysis, produced an abundance of viable tetrads. The summarized data of Table 4 include information obtained from both zero- and first-generation segregants. In all but one instance (P39-\#7-1C) the data of first-generation segregations conformed to the results expected from a consideration of the zero-generation segregations. It is clear that four of the original isolates (P39-\#6; P56-\#2, P56-\#3 and P56-\#8), and probably P39-\#7, are triploid. The data of the latter we attribute to the isolation of a rare false ascus.

The data of Table 4 suggest a method of estimating the chromosome number of $P$. tannophilus. The five zero-generation viable tetrads represent rare instances in which random meiotic assortment of chromosomes in triploid cells produced two haploid and two diploid segregants. The probability of such an event is $1 / 2^{n-1}$, where $n$ is the haploid chromosome number. The frequency of these is 5 among 107 segregations of the confirmed triploids. This value, $0 \cdot 05$, implies a haploid chromosome number of 5 . It is possible that all the isolates with trisomic markers and with low spore viability are triploids. If so, the fraction is $0 \cdot 02$, corresponding to as many as 7 chromosomes.

\section{Diploid $\times$ diploid}

Eighteen prototrophic strains were isolated from diploid $\times$ diploid mixtures (Table 1). These potential tetraploids were sporulated and subjected to genetic analysis. The results are summarized in Table 5 .

The isolates were even more disparate than were those of the haploid $\times$ diploid crosses. There were obvious differences in the segregation of the marker genes, and many of the ratios indicated diploidy, either $+/-$ or $+1+$, for specific markers. In a few instances sporulation was good but more often it was so poor that asci were difficult to find, a circumstance rare in tetraploids of $S$. cerevisiae. There were large differences in spore viability, though a general pattern was discernible; there was a weak positive correlation between spore viability and number of apparently diploid markers within an isolate. Zero-generation segregants varied widely in cell size, and diploid sporulation was infrequent among the segregants of any isolate. It was also evident that the mechanism for producing ascophores was thrown into disarray; these were often misshapen or abortive. Only eight zero-generation segregants were amenable to tetrad analysis, not more than one from any zero-generation tetrad. These segregated as normal diploids but they were too few in number to provide useful information and are not included in the data.

The random-spore data of Table 5 are not open to the clear interpretation that could be accorded the triploid data. In many instances the data are too sparse to differentiate between aneuploidy and diploidy with assurance. Furthermore, it appears likely that some of the isolates included trisomics, and the segregation expected of these, $4 \cdot 4+: 1-$, is not very different from that expected with tetravalent pairing by a tetrasomic, $3 \cdot 9+: 1-($ Roman et al., 1955). The evidence for trisomy in some isolates is to be found in the segregations for $\arg 1$ and lys 1 . For instance, the data of P45- $\$ 9$ indicate aneuploidy for argl but diploidy for lys 1 . Since these loci are linked, it seems more likely that this isolate is $+1+1-$ for $\arg 1$ and $+1-1-$ for $\operatorname{lys} 1$. 
Table 4. Confirmation of triploidy in isolates of P39 and P56 by tetrad analyses of zero- and first-generation segregants

\begin{tabular}{|c|c|c|c|c|c|c|c|c|c|}
\hline \multirow[b]{3}{*}{ Isolate } & \multirow{2}{*}{\multicolumn{4}{|c|}{ Zero-generation }} & \multicolumn{5}{|c|}{ First-generation } \\
\hline & & & & & No. of & & & & \\
\hline & Ade & Arg & Lys & Met & tetrads & Ade & Arg & Lys & Met \\
\hline P39-\#6-13A & - & + & - & - & & & & & \\
\hline B & - & + & + & - & & & & & \\
\hline $\mathrm{C}$ & + & + & + & + & 11 & $+1-$ & $+1-$ & $+1-$ & $+1+$ \\
\hline D & + & + & + & + & 14 & $+1-$ & $+1-$ & $+1+$ & $+1+$ \\
\hline P39-\#7-1A & - & + & + & - & 5 & $-1-$ & $+1-$ & $+1-$ & $-1-$ \\
\hline B & + & + & + & + & & & & & \\
\hline C & + & + & + & + & 15 & $+1-$ & $+1-^{*}$ & $+1+^{*}$ & $+1+$ \\
\hline $\mathrm{D}$ & - & - & + & + & & & & & \\
\hline P56-\#2-16A & - & + & - & - & & & & & \\
\hline B & - & - & - & + & & & & & \\
\hline $\mathrm{C}$ & + & + & + & + & 7 & $+1-$ & $+1-$ & $+1-$ & $+1+$ \\
\hline $\mathrm{D}$ & + & - & + & + & 4 & $+1-$ & $-1-$ & $+1-$ & $+1-$ \\
\hline$P 56-\# 3-13 A$ & - & - & + & + & 7 & $-1-$ & $-1-$ & $+1-$ & $+1-$ \\
\hline B & - & + & - & + & & & & & \\
\hline C & + & - & - & - & & & & & \\
\hline D & + & + & + & + & 8 & $+1-$ & $+1-$ & $+1-$ & $+1+$ \\
\hline P56-\#8-3A & - & + & + & + & 7 & $-1-$ & $+1-$ & $+1-$ & $+1+$ \\
\hline B & - & - & - & + & 8 & $-1-$ & $-1-$ & $-1-$ & $+1+$ \\
\hline C & + & + & - & - & & & & & \\
\hline $\mathrm{D}$ & + & - & + & - & & & & & \\
\hline
\end{tabular}

Table 5. Random-spore analysis of tetrad segregants of the presumed tetraploid P45, ade arg $++\mid a d e+l y s+1+$ arg + met $/++$ lys met

$+:-$ ratios that are in conflict with expectations are bracketed, with round brackets for apparent $+1-$ genotypes and square brackets for apparent $+/+$ genotypes.

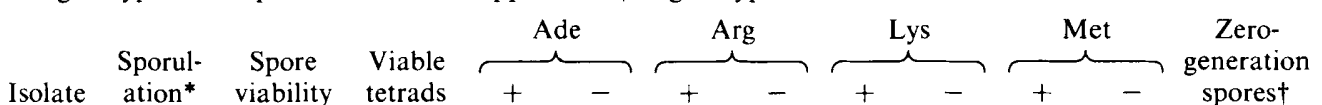

$\begin{array}{llcrrrrrrrrrl}\# 1 & \mathrm{~g} & 0 \cdot 90 & 17 / 24 & (38 & 39) & (39 & 38) & (42 & 35) & (45 & 32) & 0 \\ \# 2 & \mathrm{p} & 0 \cdot 89 & 7 / 11 & (20 & 17) & (21 & 16) & (18 & 19) & 24 & 13 & 0 \cdot 08 \\ \# 3 & \mathrm{p} & 0 \cdot 97 & 13 / 15 & (33 & 29) & (33 & 29) & (31 & 31) & {[62} & 0] & 0 \\ \# 4 & \mathrm{p} & 0 \cdot 27 & 0 / 11 & 10 & 2 & 11 & 1 & 12 & 0 & (5 & 7) & 0 \\ \# 5 & \mathrm{~g} & 0 \cdot 97 & 14 / 16 & (31 & 31) & (30 & 32) & (31 & 31) & {[62} & 0] & 0 \cdot 02 \\ \# 6 & \mathrm{p} & 0 \cdot 85 & 4 / 5 & (8 & 9) & (9 & 8) & {[17} & 0] & (9 & 8) & 0 \\ \# 7 & \mathrm{p} & 0 \cdot 60 & 3 / 16 & (21 & 17) & (23 & 15) & (19 & 18) & (21 & 17) & 0 \cdot 08 \\ \# 8 & \mathrm{p} & 0 \cdot 36 & 1 / 16 & {[23} & 0] & 17 & 6 & 14 & 9 & 17 & 6 & 0 \\ \# 9 & \mathrm{~g} & 0 \cdot 52 & 2 / 16 & 27 & 6 & 24 & 9 & (16 & 17) & (13 & 19) & 0 \cdot 03 \\ \# 10 & \mathrm{p} & 0 \cdot 33 & 1 / 15 & 15 & 6 & 16 & 5 & (12 & 9) & 15 & 6 & 0.14 \\ \# 11 & \mathrm{p} & 0 \cdot 83 & 7 / 16 & (29 & 25) & {[54} & 0] & (33 & 21) & (33 & 21) & 0 \\ \# 12 & \mathrm{p} & 0 \cdot 82 & 10 / 19 & (28 & 34) & (32 & 30) & 162 & 0] & (29 & 33) & 0 \\ \# 13 & \mathrm{p} & 0 \cdot 63 & 5 / 14 & 26 & 9 & (19 & 16) & 23 & 12 & 23 & 12 & 0.03 \\ \# 14 & \mathrm{p} & 0 \cdot 27 & 0 / 16 & 11 & 6 & 15 & 2 & 11 & 6 & 14 & 3 & 0.53 \\ \# 15 & \mathrm{p} & 0 \cdot 52 & 3 / 11 & 19 & 4 & 15 & 8 & (12 & 11) & 17 & 6 & 0.17 \\ \# 16 & \mathrm{p} & 0 \cdot 50 & 2 / 9 & (9 & 9) & (5 & 13) & (10 & 8) & 12 & 6 & 0.06 \\ \# 17 & \mathrm{p} & 0 \cdot 41 & 4 / 14 & (11 & 12) & (11 & 12) & 15 & 8 & (12 & 11) & 0 \\ \# 18 & \mathrm{~g} & 0 \cdot 47 & 2 / 16 & (14 & 16) & 24 & 6 & (14 & 16) & (16 & 14) & 0 \cdot 07 \\ \text { Total } & & & & 108 & 33 & 122 & 37 & 75 & 35 & 122 & 52 & \\ \text { Expected }(3 \cdot 9+: 1-) & & & 112 & 29 & 127 & 32 & 88 & 22 & 138 & 36 & \\ \text { Total } & & & & (242 & 238) & (222 & 209) & (238 & 216) & (183 & 162) & \\ \text { Expected }(2+: 2-) & & & (240 & 240) & (215 & 215) & (227 & 227) & (172 & 172) & \end{array}$

* g, good sporulation; p, poor sporulation.

$\dagger$ Expressed as the frequency of diploid sporulation. 
Despite such uncertainties, it is evident from the accumulated data at the bottom of Table 5 that the segregations at some loci, in particular adel and arg1, conform to those expected of tetrasomy and/or trisomy. But only one isolate, P45-\#14, is a good candidate for aneuploidy at all four loci and it may therefore be tetraploid. This isolate was also distinctive in having a very low frequency of spore germination but a relatively high frequency of diploid zero-generation segregants.

What is the origin of the non-polyploid products of a mating? There are at least four possibilities. (1) Unscheduled sporulation. The diploid parents in a mating mixture might sporulate occasionally to produce haploid segregants that subsequently participate in unscheduled matings to produce prototrophic diploids. The necessity for using YM agar as both a sporulating and mating medium would seem to invite such behaviour. A few of the isolates, as $\mathrm{P} 39-\# 3$ or P45-\#1, are indeed possible candidates for such an origin. But in other instances the occurrence of diploidy by this mechanism is unlikely; P45-\#5 is apparently $+/-$ for adel and $+/+$ for $m e t 2$, and no crosses between or within segregants of the original parental diploids can yield such a diploid. In still other instances a low frequency of spore germination suggests that some isolates which segregate as diploids are in fact aneuploid for unmarked chromosomes. (2) Reversion of a parental diploid to produce a prototrophic diploid. We have not detected such reversions among individual parental diploids. Nevertheless P45-\#5 is a candidate for such an event ; it could have arisen by reversion of P37 at the ade 1 locus. (3) Polyploid instability. Mitotic chromosome losses could lead to an array of different aneuploids. We have not tested the stability of isolates sufficiently to rule out this explanation. However, if such instability is indeed responsible for aneuploidy then most of the chromosome losses must occur prior to the original isolation of the prototrophic strains from the YM agar and not thereafter; the accumulated segregational data, particularly for adel and argl, are too consistent with theoretical expectations to permit much heterogeneity within individual isolates. (4) Incomplete karyogamy. Lagging of chromosomes at the time of fusion would lead to a diverse array of aneuploids. An indication that such losses occur has been provided by the results of the procedure used to isolate homozygous diploids (James \& Zahab, 1982); many of the supposed diploids are aberrant. However, heterozygous diploids, when produced by prototrophic selection, have provided little evidence of chromosome loss; only one of hundreds of heterozygous diploid isolates obtained in this laboratory could be characterized as possibly monosomic.

We note that prototrophic selection procedures have been used in the past to produce triploids and tetraploids in S. cerevisiae. In particular the procedure was used by Pomper et al. (1954) with results similar in many aspects to those described here. Finally, if the chromosome number of $P$. tannophilus is indeed as low as the data imply, then it is evident that with well-marked chromosomes it may be possible to identify specific chromosomes which influence ethanol production. Such chromosomes might well be useful in the construction of aneuploid strains that produce higher yields of ethanol from xylose.

This paper is NRCC Publication no. 21317.

\section{REFERENCES}

JAMES, A. P. \& ZAHAB, D. M. (1982). A genetic system for Pachysolen tannophilus, a pentose-fermenting yeast. Journal of General Microhiology 128, 2297 2301.

MaleszKa, R., James, A. P. \& Schneider, H. (1983). Ethanol production from various sugars by strains of Pachysolen tannophilus bearing different numbers of chromosomes. Journal of General Microbiology 129 , 24952500.

Pomper, S., Daniels, K. M. \& MCKeKe, D. W. (1954). (jenetic analysis of polyploid yeast. Genetici 39,343 355 .
Roman, H., Phillips, M. M. \& Sands, S. M. (1955). Studies of polyploid Saccharomyces. I. Tetraploid segregation. Genetics 40, 546561.

SCHNeIDER, H., Wang, P. Y., Chan, Y. K. \& MaleszKa, R. (1981). Conversion of D-xylose into ethanol by the yeast Pachysolen tannophilus. Biotechnology Letters 3, 8992.

Slininger, P. J., Bothst, R. J., Van Cauwenbergi, J. E. \& Kurtzman, C. P. (1982). Conversion of Dxylose to ethanol by the yeast Pachysolen tannophilus. Biotechnology and Bioengineering 24, 371-384. 